\title{
ON-CHIP PCR WITH FREE-STANDING PARYLENE CHANNEL
}

\author{
P. Satsanarukkit, H. Lo, Q. Quach, and Y.C. Tai \\ California Institute of Technology, Pasadena, CA, USA
}

\begin{abstract}
We present an on-chip PCR using a free-standing parylene channel with an integrated platinum heater for on-chip temperature cycling. The PCR chip reduces the reagent amount from tens of uL (as required by convention qPCRs) to $550 \mathrm{~nL}$. The on-chip PCR has a higher thermal efficiency than conventional PCR because of its smaller thermal capacity and good heat transfer. The demonstrated chip's free-standing channel structure reduces the thermal capacitance to $3.25 \mathrm{~mJ} /{ }^{\circ} \mathrm{C}$ and shortens the duration of PCR cycles with a thermal time constant of 3 seconds. The transparent parylene channel in visible light range enables direct optical detection. The impermeable parylene channel also prevents solution evaporation. It is shown that the pin-hole free, chemically and biologically inert parylene allows efficient PCR amplification and no additional surface treatment is required. The PCR chip can facilitate the further development of a fully-integrated, portable PCR system.
\end{abstract}

\section{INTRODUCTION}

After the discovery of PCR technique by Karis Mullis in 1986 [1], the PCR technique has become a widely-used, powerful technology for various molecular biological applications such as disease diagnosis and detection, forensic, etc.

On-chip PCR technology is particularly useful in singlecell analysis. This technology is capable of fast and easy cell loading and precise cell alignment, two critical steps in singlecell analysis. The free-standing on-chip heater and sensor reduces the system's thermal mass and increases the heating and cooling rates $[1,2]$.

Researchers have been investigating many materials for onchip PCR chambers [2]. Among inorganic materials, opaque silicon inhibits PCR amplification and bars optical detection. Though transparent, glass possibly impedes PCR reactions. Besides the less-than-satisfactory inorganic materials, researchers have looked to polymers and many have examined PDMS. However PDMS is not ideal, either. PDMS is porous and permeable, and it causes bubbles and loss of PCR samples during PCR reactions $[2,3]$. We then investigate parylene-C as the material for PCR reaction chambers and channels. Parylene is biocompatible and chemically inert. When thicker than fifteen angstroms, parylene is pin-hole free [4]. Pin-hole free parylene may reduce PCR reagent evaporation and bubble formation [2, 3]. Furthermore, parylene technology allows easy integration of other components: sample loaders, cell capturing filters, waste disposal parts, and DNA detectors [5]. A parylenebased PCR system may have less thermal mass than a PDMSbased system. In this work, we examined a PCR reaction system based on parylene-C, and we intentionally avoided any surface treatment on parylene.

For on-chip PCR amplification detection, we used a fluorescence-based detection technique. This technique includes a TaqMan ${ }^{\circledR}$ probe, which consists of a fluorophore, a quencher and a $20-40$ bp DNA single strand. In practice, the probe first binds to the amplified target DNA fragment. When DNA polymerization occurs and the polymerase cleaves the 5 ' end of the probe, the probe releases the fluorophore. Then, the released, unquenched fluorophore emits fluorescence. Because of the probe's specific binding capability, the measured fluorescence intensity is proportional to the number of the amplified target DNA fragments.

\section{FABRICATION}

The chip fabrication (Figure 1) started with DRIE etching of silicon on the back of an oxide wafer. Next, we deposited and patterned the first layer of parylene. Next, we etched the front side silicon with DRIE and $\mathrm{XeF}_{2}$ and deposited the second layer of parylene to form the channel. For the resistive heating element, a $2000 \AA$ platinum layer was deposited on the second layer of parylene and patterned using a lift off process. Then the free-standing channel was made by etching the backside of silicon wafer with DRIE and $\mathrm{XeF}_{2}$.

Figure 2 and 3 show the finished PCR chip and the final testing assembly. The parylene channel had an approximate total volume of $550 \mathrm{nl}$. The platinum heater had a resistance of $2.9 \mathrm{kOhms}$ at room temperature. A CNC-machined acrylic jig coupled the PCR chip to the loading microfluidic components.

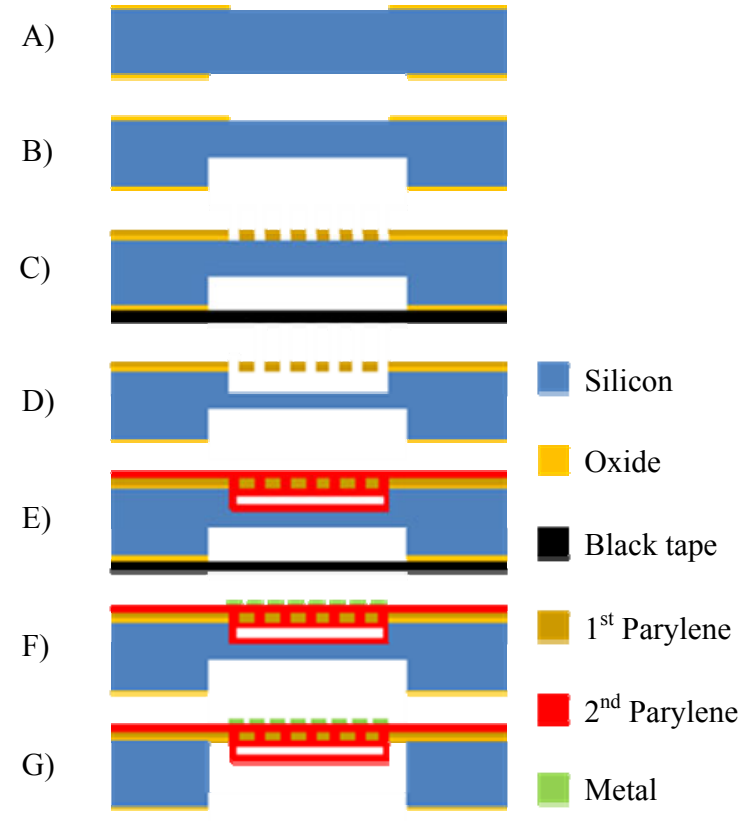

Figure 1: Chip fabrication process: A) Oxide growing and patterning; B) Backside DRIE; C) $1^{\text {st }}$ layer of parylene deposition and patterning; D) Frontside DRIE and $\mathrm{XeF}_{2}$ etching; E) $2^{\text {nd }}$ layer of parylene deposition; F) Platinum deposition and liftoff patterning; and G) Backside DRIE and $\mathrm{XeF}_{2}$ etching 


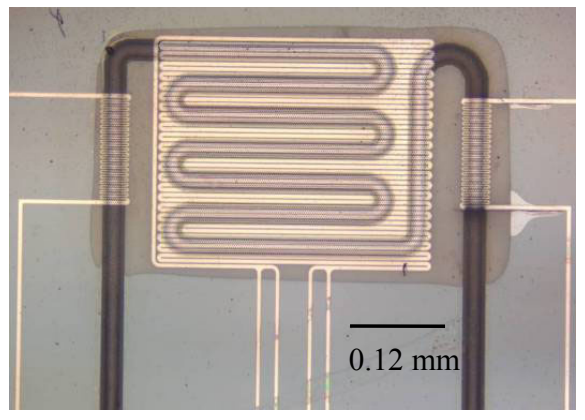

Figure 2: On-chip PCR device

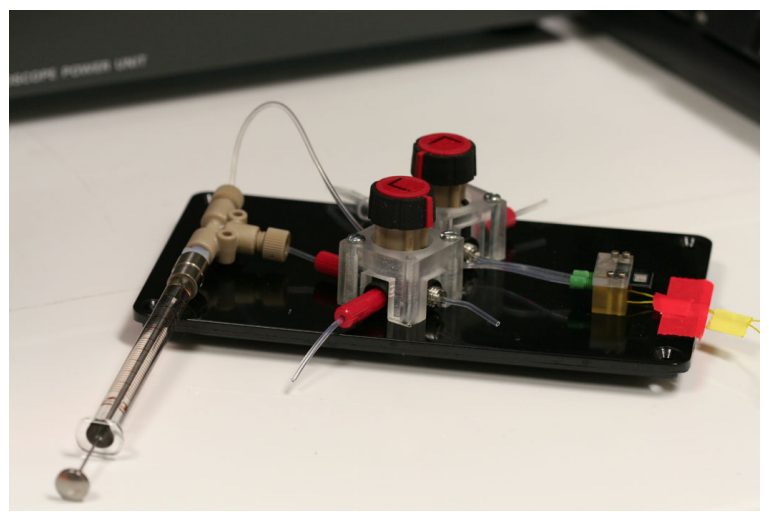

Figure 3: Loading technique using a gas-tight microsyringe manually

\section{CHIP CHARACTERIZATION}

\section{Thermal Time Constant Measurement}

To measure the heating time constant, a pulse of input power was applied, and we measured the time when the chip temperature reached $63.2 \%$ of its steady state temperature. Figure 4 shows the input power and the chip temperature profile. The chip's heating time constant is 3 seconds.

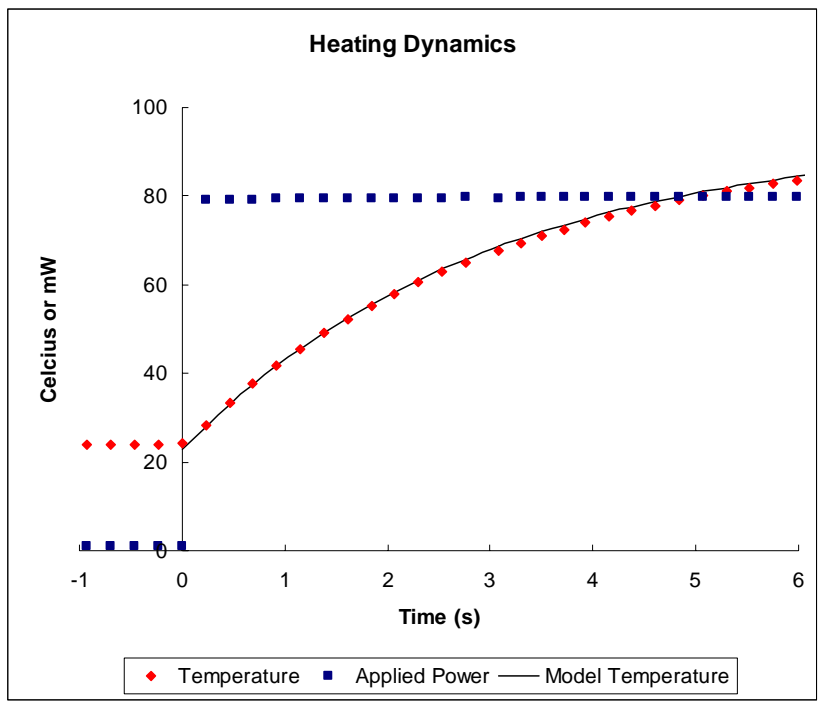

Figure 4: Chip heating time constant
To measure the cooling time constant, we first heated the chip to a certain temperature. Once the chip reached the steady state, we turned off the applied power. We measured the time when the temperature reached at $36.8 \%$ of the steady state temperature. The chip's cooling time constant is also around 3 seconds.

\section{Temperature Coefficient of Resistance}

Equation 1 shows the relation between resistance $(R)$ and temperature $(T)$ :

$$
\frac{R(T)-R\left(T_{0}\right)}{R\left(T_{0}\right)}=\alpha\left(T-T_{0}\right)
$$

where $T_{0}$ is the reference temperature, and $\alpha$ is the temperature coefficient of resistance.

To obtain the TCR, we measured the sensor's resistances at different temperatures, all of which are within the PCR's operational range. The resistance and temperature are plotted in Figure 5. The sensor's TCR is $2.0 \times 10^{-3} /{ }^{\circ} \mathrm{C}$.

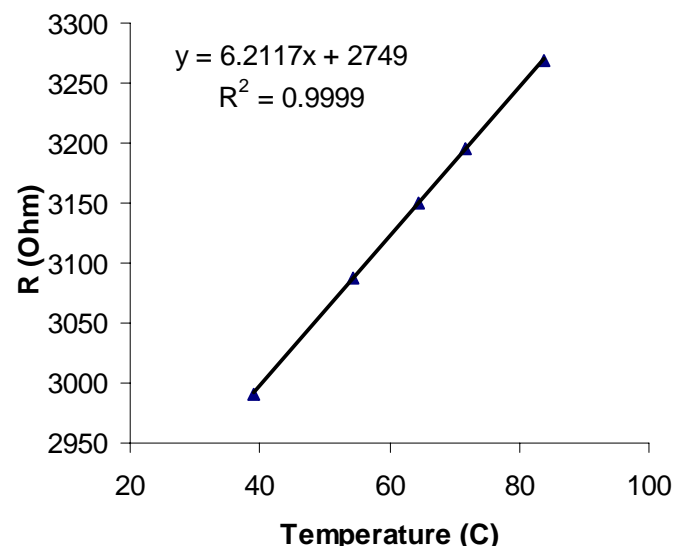

Figure 5: Temperature coefficient of resistance of the on-chip platinum sensor

\section{MATERIALS}

Parylene-C is the most commonly used among the pxylylene polymers in microfluidics due to its high mechanical strength, and fast deposition rate. Parylene-C has an USP class VI biocompatible rating. It uses chemical vapor deposition technique which operates at room temperature. The deposition first starts from dimer powder (Specialty Coating Systems) vaporizing. Then the sublimed parylene vapor is pyrolized in the pyrolysis tube at $680^{\circ} \mathrm{C}$. The obtained monomers next enter the deposition chamber and are polymerized on the surface of the substrate.

The PCR solution consists of $1 \mathrm{X}$ Quanta PerfeCTa ${ }^{\mathrm{TM}}$ MultiPlex qPCR SuperMix (Quanta Biosciences); $200 \mathrm{nM}$ of forward primer 5'-TGGAGAGGCTATTCGGCTATGACTG-3; $200 \mathrm{nM}$ of reverse primer 5'-ATACTTTCTCGGCAGGAGCA AGGTG-3'; $200 \mathrm{nM}$ of probe 5'-FAM-TAGCAGCCAGTCCC TTCCCGCTTCAGTGA-BHQ-3'(IDT), kindly designed by Arbel D. Tadmor; 1X ROX; and 6 and 90 pg of high copy plasmid bearing the ColE1 origin of replication and the kanamycin resistance gene pZS25O1+11-YFP, kindly provided by Hernan G. Garcia. The amplicon fragment is $294 \mathrm{bp}$. 


\section{EXPERIMENTAL}

First, we studied the effect of parylene on PCR efficiency. We conducted the PCR experiment with a standard thermal cycler and evaluated the results by gel electrophoresis. We compared the samples using the standard polypropylene tubes and the standard polypropylene coated with $15 \mu \mathrm{m}$ of paryleneC. In addition, we investigated the possible minimum volume of PCR reagent because we wanted to compare the effects of parylene channels and parylene-coated tube with similar SA/V ratios.

Secondly, the PCR reaction was verified with the standard qPCR machine (Stratagene Mx3000). The initial template copies varied from 10 to $10^{7}$ copies with tenfold increment. The total volume is limited to $20 \mu \mathrm{L}$ each tube because of the machine's specifications.

Then, we conducted the on-chip PCR reaction. We first cleaned the parylene channel with DNA decontamination solution (Ambion) and rinsed with DEPC- treated and sterile filtered water (Sigma Aldrich). We manually injected the fluids with a micro syringe (Hamilton), as shown in Figure 3. All of the loading procedures do not need any bulky external pumping system, leading to feasibility to be the portable device. Then the device was filled with the PCR solution described above. Approximately $550 \mathrm{nl}$ of PCR mixture was injected.

For thermal cycling, the on-chip platinum resistor was used as both the heater and temperature sensor. The voltage was supplied from Universal Source (HP 3245A) to the chip and the current was measured with a precision multimeter (Agilent 34401A). The close-loop temperature control for PCR thermal cycling was done with a LabView PID feedback control program. The PCR thermal cycling started with $95^{\circ} \mathrm{C}$ for 3 minutes, followed by 50 cycles of $95^{\circ} \mathrm{C}$ for 15 seconds (denaturation) and $60^{\circ} \mathrm{C}$ for 90 seconds (annealing/extension) respectively. Figure 6 and Figure 7 show the first 3 cycles of input power and temperature profiles.

To measure fluorescence, fluorescent images were taken after each thermal cycle using a Nikon Eclipse E800 fluorescence microscope. The E800 microscope has a Nikon super high pressure mercury lamp power supply (Nikon Inc.), and an integrated CCD camera. Figure 8 shows the testing assembly and the fluorescent microscope. The fluorescence picture was then analyzed with image processing software, Image $J$ (National Institutes of Health). To reduce the noise from fluctuation of light source and autofluorescence of the chip during the measurement, the obtained fluorescence intensity was normalized against the parylene background on the base of the chip.

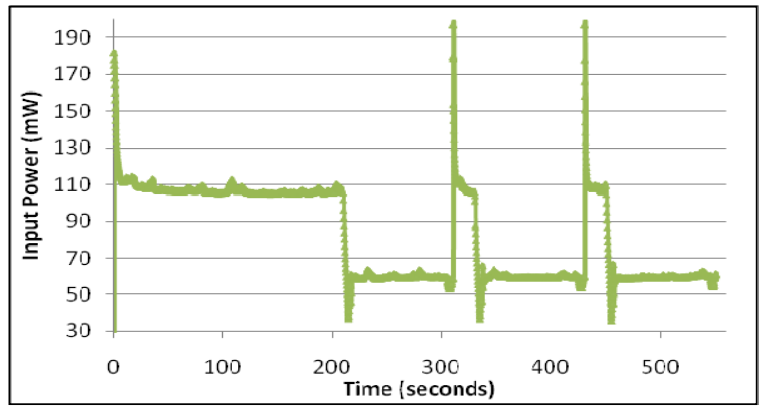

Figure 6: Input power of the initiation and the first 3 thermal cycles

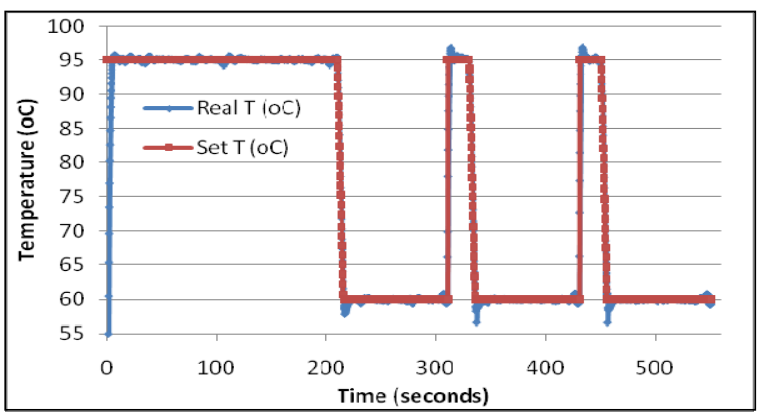

Figure 7: Temperature profiles of the initiation and the first 3 thermal cycles

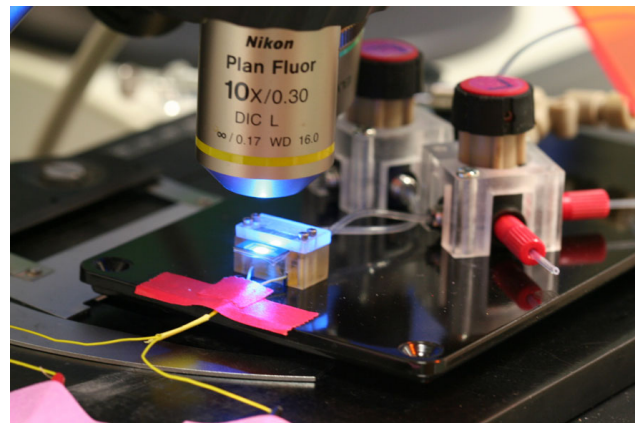

Figure 8: Fluorescence image taking at the end of each thermal cycle

\section{RESULTS AND DISCUSSIONS}

Figure 9 shows the gel electrophoresis images of the PCR experiments using a standard thermal cycler. In Figure 9, the amplicon fragments of $294 \mathrm{bp}$ were shown with the $100 \mathrm{bp}$ DNA ladder on the left. The minimum volume for distinguiable bands was $2 \mu \mathrm{L}$. No primer-dimer was observed. Also, the parylene coated tubes gave good amplification results. The notemplate-control (NTC) tube shows no amplification band.

A)

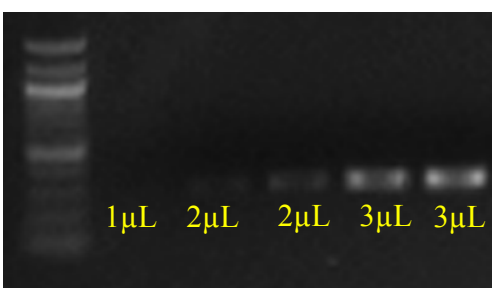

B)

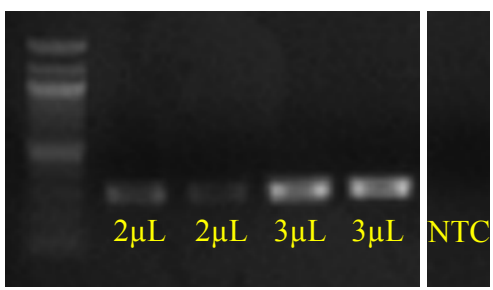

Figure 9: Gel electrophoresis images: A) Standard polypropylene tubes of volume 1, 2, 2, 3, and $3 \mu \mathrm{L}$; and B) Standard polypropylene coated with $15 \mu \mathrm{m}$ of parylene- $C$ of volume 2, 2, 3, $3 \mu \mathrm{L}$ and No Template Control (NTC) using a standard thermal cycler 
Figure 10 shows the normalized amplification plots from the standard qPCR machince (Stratagene Mx3000). The resulting plot has the same curves as standard plots. The results also show that the probe and other PCR reagent were working well.

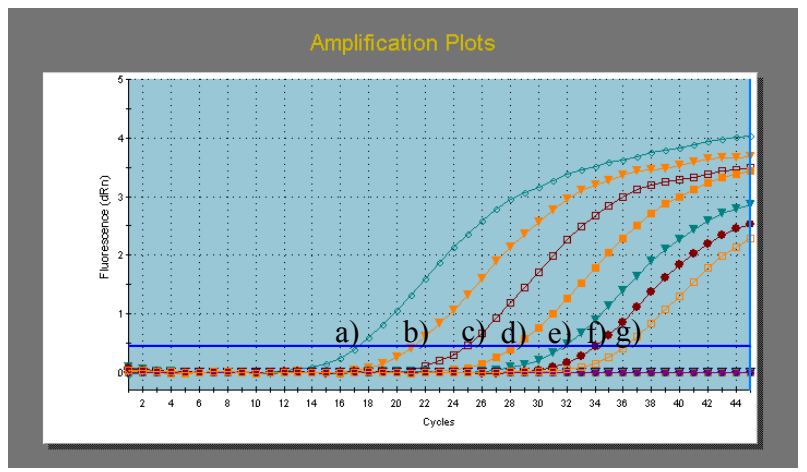

Figure 10: Normalized amplification plots of initial copies of templates of a-f: a) $10^{7}$; b) $10^{6}$; c) $10^{5}$; d) $10^{4}$; e) $10^{3}$; f) $10^{2}$; and g) 10 using a standard qPCR machine

For the on-chip PCR results, Figure 11 shows the channel fluorescence pictures of $90 \mathrm{pg}$ initial template after the thermal cycles $0^{\text {th }}, 10^{\text {th }}, 20^{\text {th }}, 30^{\text {th }}, 40^{\text {th }}$ and $50^{\text {th }}$ respectively. The intensity successfully increases over the cycles. Figure 12 shows the comparison of amplification plots of fluorescence $(\mathrm{dRn})$ of 6 and $90 \mathrm{pg}$ initial templates, and the no primer control (NPC). These results show that the templates were significantly amplified within the first few PCR cycles. In addition, the curves clearly show the differences of different initial template amounts respectively. This device can differentiate different starting template quantities.

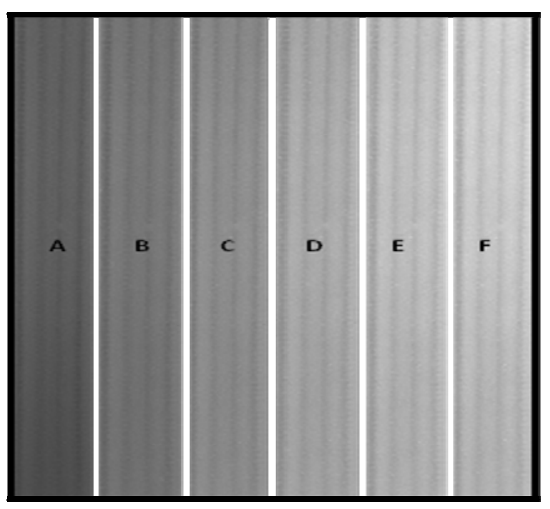

Figure 11: On-chip fluorescence images of the $90 \mathrm{pg}$ initial template after cycle: A) $0^{\text {th }}$ B) $10^{\text {th }}$ C) $20^{\text {th }}$ D) $30^{\text {th }}$ E) $40^{\text {th }}$ F) $50^{\text {th }}$

We are improving device designs. The presented chip cannot achieve uniform temperature distribution across the chip. Sporadically, bubbles appear in the channel and impede PCR efficiency. Future chips will incorporate heaters with optimized geometry that improves on-chip heat distribution. Changing PCR preparation protocols may reduce bubble generation. More work is underway.

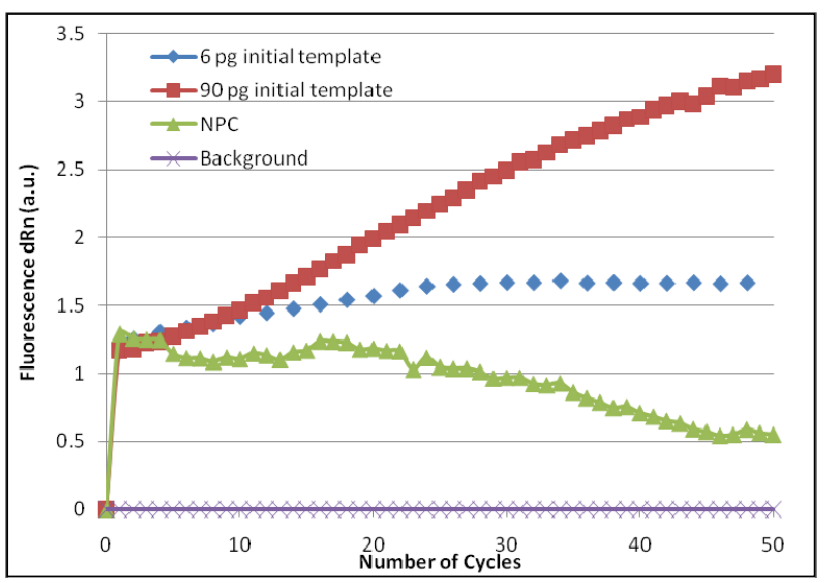

Figure 12: On-chip fluorescence intensities: 1) 6 pg initial template 2) $90 \mathrm{pg}$ initial template 3) No Primer Control

\section{CONCLUSIONS}

In conclusion, a free-standing parylene-C PCR channel with an on-chip Pt heater/sensor for fast thermal cycling was demonstrated. Fast cycling and efficient PCR amplification were observed. The device can distinguish different initial amount of templates. This work validates that parylene-C is an adequate material for on-chip PCR and further downsizing of the device to reduce its volume and integrating necessary microfluidic components are also possible. However, the heating uniformity and bubble suppression should still be improved.

\section{ACKNOWLEDGEMENTS}

This work was supported by The Royal Thai Government scholarship and The Boeing Company. The authors would like to thank Dr. Franz M. Weinert, Peera Jaru-Ampornpan, Kuang Shen, Arbel D. Tadmor, and Hernan G. Garcia for all the PCR trainings, sample preparations and valuable technical support. The authors appreciate all generous help and precious advice from the members of Caltech Micromachining Laboratory.

\section{REFERENCES}

[1] A. J. Mello, "DNA Amplification: Does 'Small' Really Mean 'Efficient'?”, Lab On a Chip, 1 (2001), pp. 24N-29N.

[2] C. Zhang and D. Xing, "Miniaturized PCR Chips for Nucleic Acid Amplification and Analysis: Latest Advances and Future Trends", Nucleic Acids Research, 35, 13 (2007), pp. 4223-4237.

[3] Y. S. Shin, K. Cho, S. H. Lim, S. Chung, S. J. Park, C. Chung, D. C. Han and J. K. Chang, "PDMS-based Micro PCR Chip with Parylene Coating", J. Micromech. Microeng. 13 (2003), pp. 768-774.

[4] J. J. Senkevich and P. I. Wang, "Molecular Layer Chemistry via Parylenes", Chemical Vapor Deposition, 15 (2009), pp. 91-94.

[5] S. Sukas, A. E. Erson, C. Sert, and H. Kulah, "A Parylenebased Dual Channel Micro-Electrophoresis System for Rapid Mutation Detection via Heteroduplex Analysis", Electrophoresis, 29 (2008), pp. 3752-3758.

\section{CONTACT}

*P. Satsanarukkit, tel: +1-626-395-3885; penvipha@mems.caltech.edu 